\title{
A Distributed Clustering Methodology for Connecting Dense Sensor Network Fields
}

\author{
Hamid Ali Abed Alasadi, Ali A. Yassinand Abdullah Mohammed Rashid \\ Computer Science Department, \\ Faculty of Education for Pure Science, Basra University, Basra, Iraq
}

\begin{abstract}
Clustering is a blooming topology control methodology, which can extend the lifetime and upturn scalability for wireless sensor networks. The admired principles for clustering procedure are to select cluster heads with additional residual energy and to interchange them occasionally. Sensors at substantial traffic locations swiftly exhaust their energy resources and expire much earlier, leaving behind energy hole and network barrier. Clustering goes behind some advantages like network scalability, localizing route setup, using communication bandwidth efficiently and takes the advantage of network lifetime. By the data aggregation procedure, needless communication between sensor nodes, cluster head and the base station is eluded. An evaluation of energy efficient optical low energy adaptive clustering hierarchy $(O-L E A C H)$ has been performed and the enactments have been compared with the prevailing low energy adaptive clustering hierarchy $(L E A C H)$ algorithm, between two detached wireless sensor network fields. O-LEACH procedure has been primarily implemented to join two distinct wireless sensor fields. An optical fiber is used to join two reserved wireless sensor fields. This distributed clustering methodology chiefly targets in exploiting the parameters like network lifetime, throughput and energy efficiency of the whole wireless sensor system.
\end{abstract}

Keywords: Distributed clustering algorithm, wireless sensor network, energy efficiency, clustering, network lifetime.

\section{INTRODUCTION}

Wireless sensor network (WSN) is main and very motivating technology applied to diverse applications like observing the accessible conditions in specific areas. Each sensor node contains a wireless transceiver, a microcontroller and a battery. The foremost advantages of these networks are self-organization, fault tolerance characteristics, energy efficiency, avoiding wiring problems and being accessed through a centralized control. In order to drop the data transmission time and energy consumption, the sensor nodes are assembled into numerous clusters. The assemblage of sensor nodes is recognized as clustering. In cluster construction, every cluster has a leader which is identified as cluster head. A cluster head is one of the sensor nodes which have unconventional capabilities than other sensor nodes. The cluster head is nominated by the sensor nodes in the pertinent cluster and may also conceivable by the user to pre-assign the cluster heads. The cluster head is used to communicate the aggregated data to the sink or base station. The rest of this paper has been prearranged as follows. Low Energy Adaptive Clustering Hierarchy (LEACH) clustering methodology is discussed in section II. Section III gives the conceptual description and review of a well-distributed clustering algorithm, the optical low energy adaptive clustering hierarchy (O-LEACH). Simulation results have been briefed in section IV. Section V concludes the paper and gives few ideas for an improvement over the existing algorithm.

\section{Literature Survey of Existing Algorithms}

Distributed clustering is extensively used in sensor node grouping since it provides improved data collection and reliability [1].LEACH is a clustering mechanism that distributes energy consumption all alongside its network, the network being alienated into clusters, $\mathrm{CHs}$ which are virtuously distributed in manner and the randomly selected $\mathrm{CHs}$ assemble the data from the nodes which are coming under its cluster [2]. LEACH forms clusters by using a distributed algorithm, where nodes make independent decisions without any centralized control [3]. The key disadvantage of this algorithm is that, when a random node nominated as cluster head dies during its operation, the 
complete cluster becomes dysfunctional thereby not appropriate for large-scale wireless sensor network[4-6].LEACH is a clustering mechanism that distributes energy consumption all along its network, the network being alienated into clusters and the CHs are purely distributed in manner, and the randomly selected $\mathrm{CHs}$ gather the information from the nodes which are imminent under its cluster[7]. LEACH protocol includes four main steps for each round: the advertisement phase, the cluster set-up phase, the schedule creation and the data transmission. Throughout the advertisement phase, the appropriate $\mathrm{CH}$ nodes will be distributing declaration to the nodes a waiting under them to turn out to be a member in its cluster [8-10]. The sensor nodes will accept the offer based on Received Signal Strength (RSS). In the cluster set-up phase, the nodes will be answering to their selected CHs. In schedule creation step, as the $\mathrm{CH}$ receives answer from the nodes, it has to create a Time Division Multiple Access (TDMA) schedule and send back to its cluster members to intimate them when they have to permit the information to it. In the data transmission step, the data collected by the specific sensors will be given to the cluster head throughout their time intervals [11]. The chiefrestriction here is that, the cluster member nodes will be turned off to decrease energy consumption after the data transmission during particular time slot gets finished [12].

\section{The O-LeACH Algorithm}

In O-LEACH algorithm, the construction of the sensor network encompasses of a distributed fiber sensor link and two detached wireless sensor network fields. Figure 1, displays the disposition of sensor nodes in the two fields. The Distributed Fiber Sensor (DFS) link is positioned at the center of the sensor fields and can cover a certain area. The two WSN fields are occupied with randomly scattered nodes and these nodes can or cannot communicate with each other liable on the required applications. Since the DFS offer data processing, at one end of the DFS link, the base station for all the sensor nodes is situated.

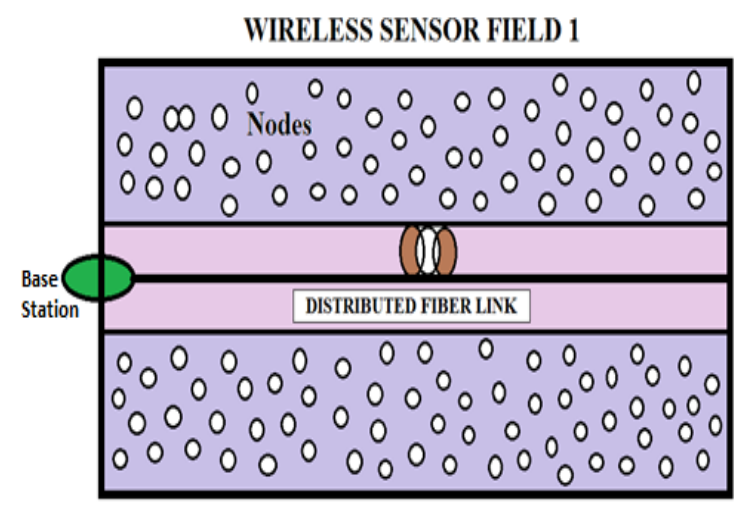

WIRELESS SENSOR FIELD 2

Figure1. Two Separate Sensor fields connected using a DFS link

Although the lifetime of the DFS may have to be deliberated as well, the lifetime of such a sensor network is largely determined by the WSN. It consequences that the clustering based algorithm still displays good energy efficiency in the sensor network, particularly when two WSNs are not accessible to each other. As the DFS shelter extended rectangular area, O-LEACH algorithm is used to examine the lifetime of the hybrid sensor network with rectangular topology by isolating and connecting a series of WSNs around the DFS.

The $\mathrm{CH}$ nodes aggregates the arriving data from the nodes that fit to the suitable cluster and propel the aggregated packet to the base station in order to furthermore trim down the amount of data that must be forwarded to the base station, thereby augmenting the system lifetime. Succeeding to a given interval of time, to adjust the uniformity of energy dissipation of the network, randomized rotation of the concern of cluster head is carried out. Sensors elect themselves to be local cluster heads at any precise time with a definite probability.

As the operation of the standard LEACH algorithm is separated into setup phase and steady phase, OLEACH operation is likewise separated into two phases, and the steady phase is as same as LEACH. During the setup phase, the selection of cluster head follows the analogous criteria as LEACH, but there are two major alterations between O-LEACH and LEACH: (i) Nodes in WSNs cannot be deployed in the DFS coverage area and (ii) The cluster head and the node must be within the same WSN field if two WSNs cannot communicate with each other. 
For most applications, it is superior to assume that the two WSN fields are isolated due to the following reasons: (i) Lengthier data transfer distance over the DFS terrain ends with advanced energy consumption and (ii) Wireless communication over the DFS area is not even permitted for some applications.

Table1. Simulation Parameter Setup

\begin{tabular}{|c|c|}
\hline Parameter & Values \\
\hline Topology & $500 \times 500 \mathrm{~m}^{2}$ \\
\hline Number of nodes & 30 \\
\hline Data packet size & 4000 bytes \\
\hline Control packet size & 550 bytes \\
\hline Initial energy & 1 Joule \\
\hline Transmitter power & $31.32 \mathrm{~mW}$ \\
\hline Receiver power & $35.28 \mathrm{~mW}$ \\
\hline Ideal power & $712 \mathrm{~mW}$ \\
\hline Sleep power & $144 \mathrm{~mW}$ \\
\hline
\end{tabular}

\section{Simulation Results}

The O-LEACH algorithm has been simulated using the network simulator (NS-2). Table 1, displays the necessary simulation parameter setup. In O-LEACH, the base station is expected to be situated at the center of the left edge of the two wireless sensor fields. The network energy dissipation is absolutely an arithmetical behaviour due to the random distribution of WSN nodes. As the DFS is absolutely active, the lifetime of the DFS is not deliberated. Figure 2, demonstrates the network performance in terms of the percentage lifetime of O-LEACH and LEACH. Initially in 100 rounds, the percentage lifetime is 90 for both the algorithms. In 500 rounds, the percentage lifetimes of OLEACH and LEACH are 72 and 53 respectively. Similarly in 3000 rounds, the percentage lifetimes of $\mathrm{O}-\mathrm{LEACH}$ and $\mathrm{LEACH}$ are 14 and 1 respectively.

Table2. Percentage lifetime for LEACH and $O-L E A C H$

\begin{tabular}{|c|c|c|c|c|c|c|c|c|}
\hline \multicolumn{2}{|c|}{ Number of Rounds } & 100 & 500 & 1000 & 1500 & 2000 & 2500 & 3000 \\
\hline \multirow{2}{*}{ } & 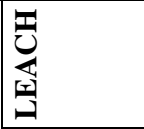 & 90 & 53 & 40 & 20 & 4 & 2 & 1 \\
\hline & 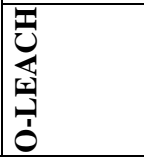 & 90 & 72 & 62 & 43 & 24 & 20 & 14 \\
\hline
\end{tabular}

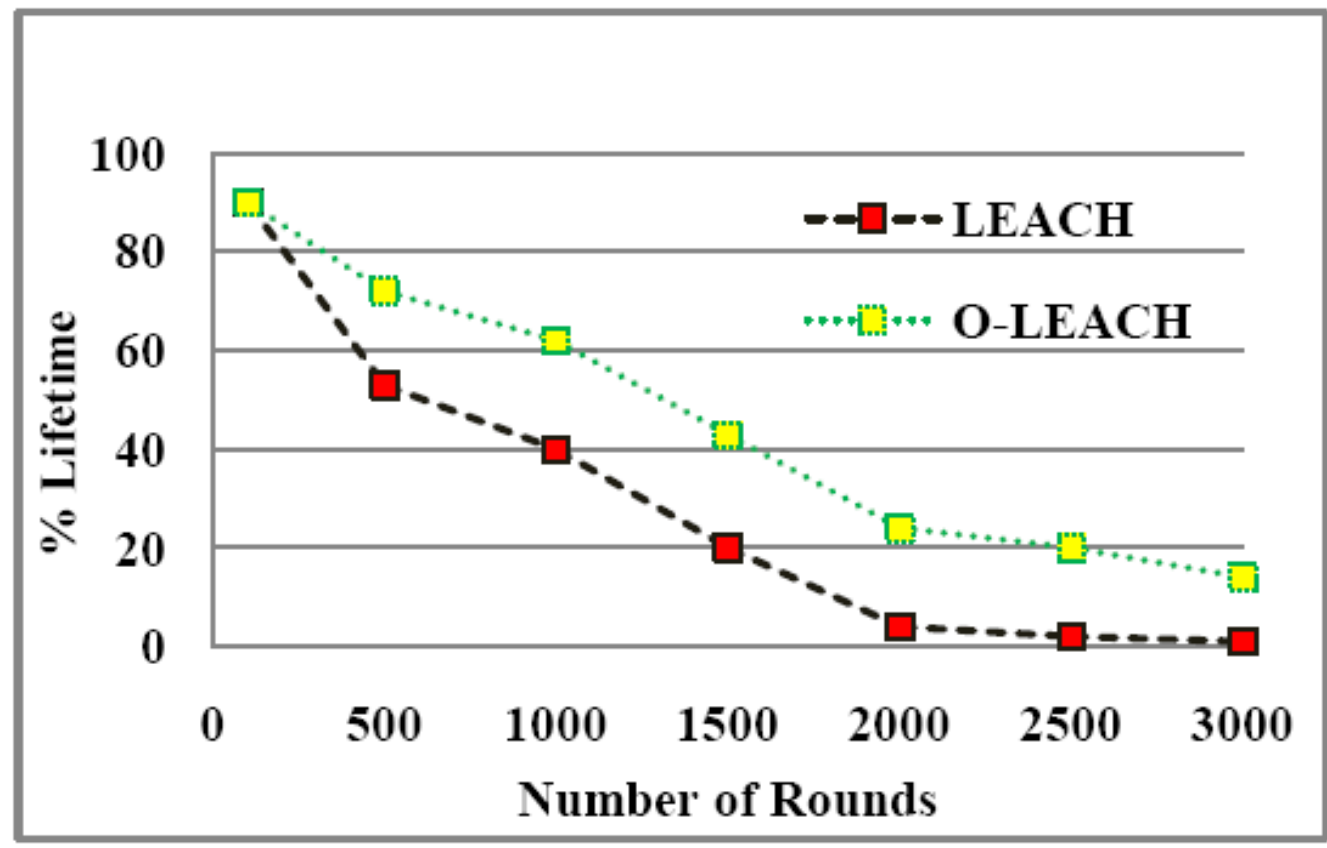

Figure2. Percentage Lifetime versus Number of Rounds 


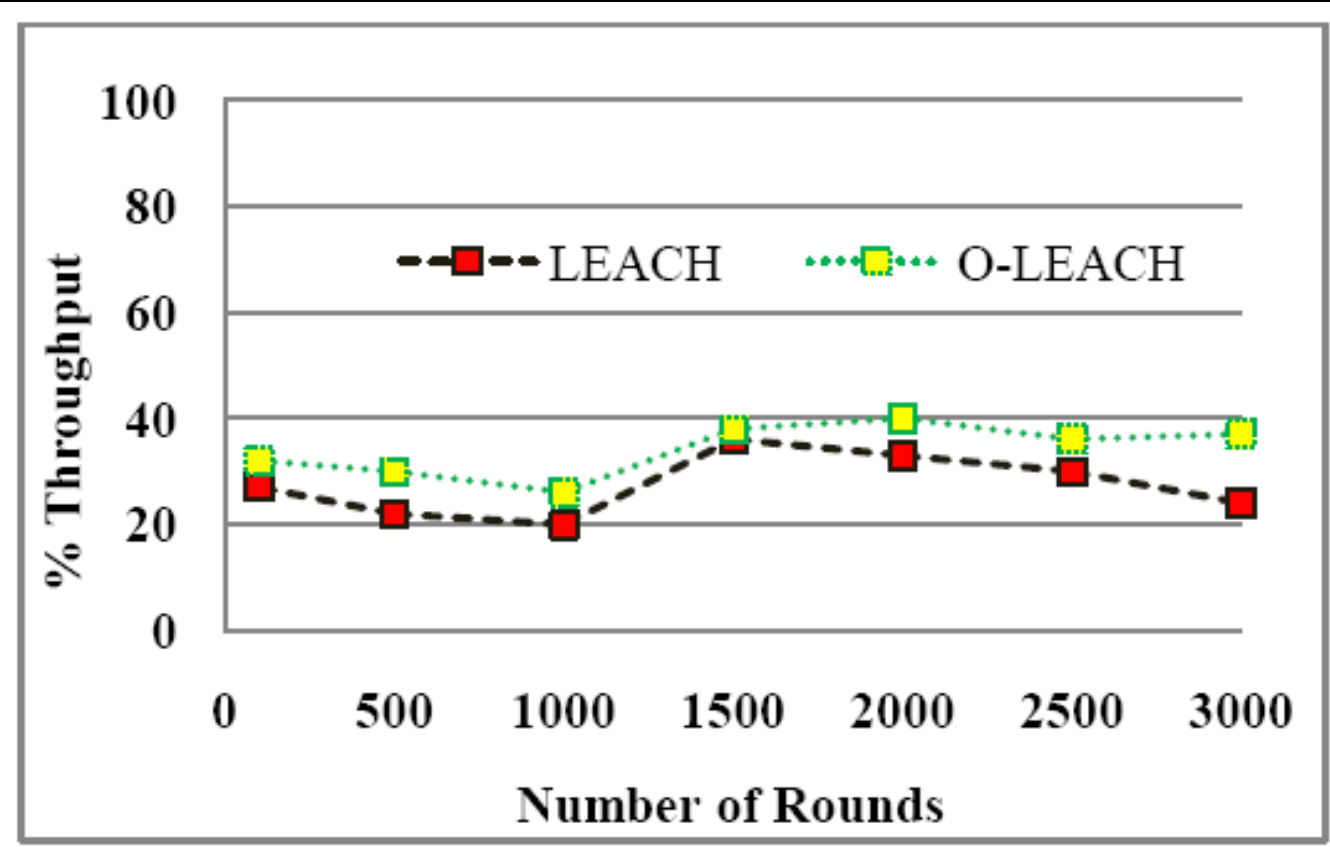

Figure3. Throughput versus Number of Rounds (O-LEACH and LEACH)

Table3. Percentage throughput for LEACH and O-LEACH

\begin{tabular}{|c|c|c|c|c|c|c|c|c|}
\hline \multicolumn{2}{|c|}{ Number of Rounds } & 100 & 500 & 1000 & 1500 & 2000 & 2500 & 3000 \\
\hline \multirow{2}{*}{ 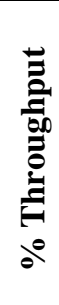 } & 矛 & 27 & 22 & 20 & 36 & 33 & 30 & 24 \\
\hline & 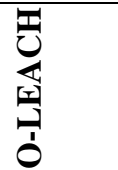 & 32 & 30 & 26 & 38 & 40 & 36 & 37 \\
\hline
\end{tabular}

The percentage lifetime is found to be linearly increased in O-LEACH, when compared to LEACH. This is because of the usage of DFS link for connecting the sensor fields, which greatly reduces the losses while routing the packets to the base station. It is to be noted that in most practical applications, the DFS coverage is very specific and cannot be freely varied. Sometimes, parallel DFS links may be deployed to cover more broad areas. Figure 3, shows the performance evaluation of throughput and number of rounds for the two algorithms. Initially in 100 rounds, for O-LEACH and LEACH, the percentage of throughput is 32 and 27 respectively. Similarly in 3000 rounds, the throughput is 37 and 24 for O-LEACH and LEACH respectively. This slight increase in throughput in O-LEACH is mainly due the usage of DFS link for data packet transmission to the base station. Figure 4, shows the energy efficiency comparison of the two algorithms. After 100 rounds, the energy efficiency is found to be improved linearly in O-LEACH when compared to LEACH, for every successive rounds. This clearly indicates that, O-LEACH is efficient mainly when two wireless sensor networks have to be connected together.

Table4. Energy Efficiency for LEACH and $O$-LEACH

\begin{tabular}{|c|c|c|c|c|c|c|c|c|}
\hline \multicolumn{2}{|c|}{ Number of Rounds } & 100 & 500 & 1000 & 1500 & 2000 & 2500 & 3000 \\
\hline \multirow{2}{*}{ 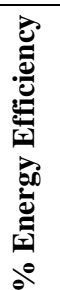 } & 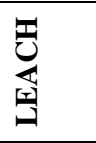 & 42 & 45 & 43 & 46 & 38 & 27 & 20 \\
\hline & 全 & 40 & 35 & 30 & 17 & 12 & 8 & 5 \\
\hline
\end{tabular}




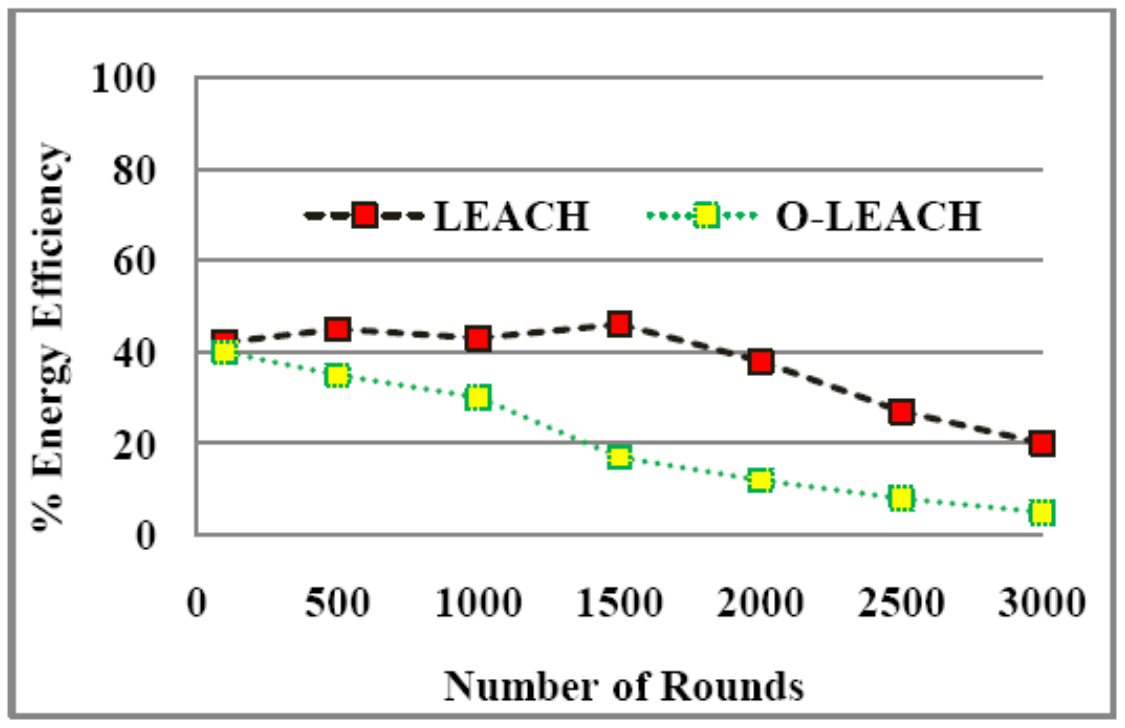

Figure4. Energy efficiency versus Number of Rounds (O-LEACH and LEACH)

\section{Conclusion And Future Directions}

Clustering is the most significant issue for networks where resources are restricted. Thus, it is required to make use of the available energy efficiently. There becomes a need in wireless sensor network to effectively group the sensor nodes in to clusters. In this paper, a review of O-LEACH algorithm has been carried out and the performance evaluation has been done against the existing LEACH algorithm, between two separate wireless sensor network fields. It could be clearly seen that the performance of O-LEACH is comparatively better, thereby O-LEACH is efficient mainly when two separate wireless sensor networks have to be connected together. The optical fiber links leads to various disadvantages like: i) Scattering losses, it is not applicable to use the DFS link for efficient long haul communications, ii) The changes in the light properties usually happen in the sensor, iii) Need for more expensive optical transmitters and receivers, iv) More difficult and expensive to employ splices and v) Difficult to carry electrical power to operate the terminal devices. These drawbacks could be overcome by changing the DFS link with an alternate medium for communication and effectively connecting two separate wireless sensor fields.

\section{REFERENCES}

[1] Akyildiz, I, Su, W, Sankarasubramaniam, Y \&Cayirci, E 2002, 'A survey on sensor networks', IEEE Communications Magazine, vol. 40, no. 8, pp. 102-114.

[2] Ali, M, Voigt, T \&Uzmi, ZA 2006, 'Mobility management in sensor networks', Proceedings of the 2nd International Conference on Distributed Computing in Sensor Systems, pp. 131-140.

[3] Baranidharan, B \&Shanthi, B 2010, 'A survey on energy efficient protocols for wireless sensor networks', International Journal of Computer Applications, vol. 11, no. 10, pp. 0975-8887.

[4] Guo, L, Xie, Y, Yang, C \& Jing, Z 2010, 'Improvement on LEACH by combining adaptive cluster head election and two-hop transmission', Proceedings of the 9th International Conference on Machine Learning and Cybernetics, pp. 11-14.

[5] Haitao, Z, Shiwei, Z \&Wenshao, B 2014, 'A clustering routing protocol for energy balance of wireless sensor network based on simulated annealing and genetic algorithm', International Journal of Hybrid Information Technology, vol. 7, no. 2, pp. 71-82.

[6] Han, L 2010, 'LEACH-HPR: An energy efficient routing algorithm for heterogeneous WSN', Proceedings of the IEEE International Conference on Intelligent Computing and Intelligent Systems, pp. 507-511.

[7] Heinzelman, WB, Chandrakasan, AP \& Balakrishnan, H 2002, 'Application specific protocol architecture for wireless micro sensor networks', IEEE Transactions on Wireless Communications, vol. 1, no. 4, pp. 660-670.

[8] Hill, J 2000, 'System architecture directions for networked sensors', Proceedings of the 9th International Conference on Architectural Support for Programming Languages and Operating Systems, pp. 93-104. 
[9] Hossein, J 2013, 'An introduction to various basic concepts of clustering techniques on wireless sensor networks', International journal of Mobile Network Communications and Telematics, vol. 3 , no. 1, pp. 01-17.

[10] Huifang, C, Hiroshi, M, Yoshitsugu, O, Tomohiro, K \&Tadanori, M 2007, 'Adaptive data aggregation for clustered wireless sensor networks', Proceedings of the Ubiquitous Intelligence and Computing, pp. 475-484.

[11] Jaswant, SR, Neelesh, G \& Neetu, S 2014, 'Energy efficient data communication approach in wireless sensor networks', International Journal of Advanced Smart Sensor Network Systems, vol. 4, no. 3, pp. 01-12.

[12] Jeong, H, Nam, CS, Jeong, YS \& Shin, DR 2008, 'A mobile agent based LEACH in wireless sensor network', Proceedings of the International Conference on Advanced Communication Technology, pp. 75-78. 